\title{
Mechanisms of elongation on the ribosome: dynamics of a macromolecular machine
}

\author{
W. Wintermeyer"1, F. Peske", M. Beringer†, K.B. Gromadski†, A. Savelsbergh" and M.V. Rodnina† \\ "Institute of Molecular Biology, University of Witten/Herdecke, Stockumer Str. 10, 58448 Witten, Germany, and †Institute of Physical Biochemistry, \\ University of Witten/Herdecke, Stockumer Str. 10, 58448 Witten, Germany
}

\begin{abstract}
Protein synthesis in the cell is performed on ribosomes, large ribonucleoprotein particles, which in bacteria consist of three RNA molecules and over 50 proteins. This review summarizes recent progress in understanding the mechanisms of the elongation phase of protein synthesis. Results from rapid kinetic analysis of elongation reactions are discussed in the light of recent structural data.
\end{abstract}

The ribosome is a macromolecular machine that synthesizes proteins from aa-tRNA (aminoacyl-tRNA) according to the sequence encoded in mRNA. The elongation phase of protein synthesis is a cyclic process consisting of three basic steps. First, aa-tRNA carried by EF-Tu (elongation factor Tu) binds to the A site of the ribosome. Secondly, A-site-bound aa-tRNA reacts with P-site-bound pept-tRNA (peptidyltRNA) to form a peptide bond, resulting in deacylated tRNA in the $\mathrm{P}$ site and pept-tRNA prolonged by one amino acid in the A site. Thirdly, the cycle is completed by the translocation of pept-tRNA from the A site to the $\mathrm{P}$ site; during the movement, the mRNA is carried along with the tRNA, and deacylated tRNA dissociates from the $\mathrm{P}$ site via the exit (E) site. The elongation cycle is repeated until the entire coding sequence of the mRNA is translated and a termination codon appears in the decoding site, whereupon translation is terminated. All phases of ribosomal protein synthesis, except for peptide-bond formation itself, are catalysed by translation factors, some of which are GTPases that hydrolyse GTP during their functional cycles.

In recent years, enormous progress has been made in structure determination of the components of the translation apparatus, including atomic structures of many translation factors and both subunits of the bacterial ribosome. In parallel, functional studies using rapid kinetic analysis have provided kinetic mechanisms of the partial reactions of the elongation cycle in detail. A recurrent theme that emerges from these studies is that induced conformational changes of the ribosome form essential elements of the functional mechanism. The aim of this review is to summarize the recent biochemical and kinetic evidences and discuss them in relation to the structural information. Detailed reviews on ribosome structures and on the initiation and termination of translation have appeared recently [1-10].

Key words: elongation factor, mRNA decoding, peptide-bond formation, rapid kinetics, translocation.

Abbreviations used: aa-tRNA, aminoacyl-tRNA; EF-G, elongation factor $G$; EF-Tu, elongation factor Tu; pept-tRNA, peptidyl-tRNA; PT, peptidyl transferase.

${ }^{1}$ To whom correspondence should be addressed (email winterme@uni-wh.de).

\section{Aa-tRNA selection: the importance of induced fit}

The ribosome recognizes aa-tRNAs according to the match between anticodon and mRNA codon in the $\mathrm{A}$ site. AatRNA binds to the ribosome in a ternary complex with EFTu and GTP (Figure 1). After codon-anticodon recognition, a conformational change in EF-Tu is induced that leads to GTPase activation. GTP hydrolysis causes a major conformational change of EF-Tu, which triggers the release of aa-tRNA and the accommodation of the $3^{\prime}$ end in the PT (peptidyl transferase) centre on the 50S subunit, followed by peptide-bond formation [11-13].

The fidelity of aa-tRNA discrimination is high, with less than 1 incorrect out of 1000 correct amino acids incorporated into a peptide. Incorrect aa-tRNAs are rejected at two stages, which are separated by GTP hydrolysis: during initial selection of ternary complexes and during proofreading of aa-tRNA. The ribosome contributes to selection in that it both enhances the stabilities of correct codonanticodon duplexes and accelerates the forward rates of GTPase activation and accommodation of correct aa-tRNAs, implicating duplex stability and induced fit as sources of selectivity $[14,15]$. Under conditions where the fidelity of tRNA selection was high and close to that found in vivo, the dissociation rate constants of cognate and near-cognate ternary complexes differed by a factor of 400 . The rate of GTP hydrolysis in the cognate ternary complex was $250 \mathrm{~s}^{-1}$, and was strongly decreased to $0.4 \mathrm{~s}^{-1}$ by a C-A mismatch at the first position. It is important to note that the large stability difference between cognate and near-cognate codonanticodon complexes does not contribute to initial selection, since A-site binding is a non-equilibrium process that is driven by the irreversible forward reaction of GTP hydrolysis, resulting in a decrease in apparent affinity $K_{\mathrm{M}}$ for the cognate substrate. Owing to the large differences in GTPase rates, $K_{\mathrm{M}}$ values are higher for cognate substrates than for near-cognate substrates; hence, there is essentially no discrimination on the basis of substrate binding. Consequently, the selectivity of the initial selection step is kinetically controlled and is solely due to the much faster (650-fold) GTP 
Figure 1| Mechanism of tRNA selection

Kinetic scheme of EF-Tu-dependent aa-tRNA binding to the ribosomal A site. Kinetically resolved steps are indicated by numbered rate constants; chemical steps that are intrinsically rapid and rate-limited by the preceding step are designated $k_{\text {GTP }}$ and $k_{\text {pep. }}$. EF-Tu is depicted in different conformations in GTP- and GDP-bound states and in the activated GTPase state. Four rate constants, $k_{-2}, k_{3}, k_{5}$ and $k_{7}$, contribute to tRNA discrimination [11].
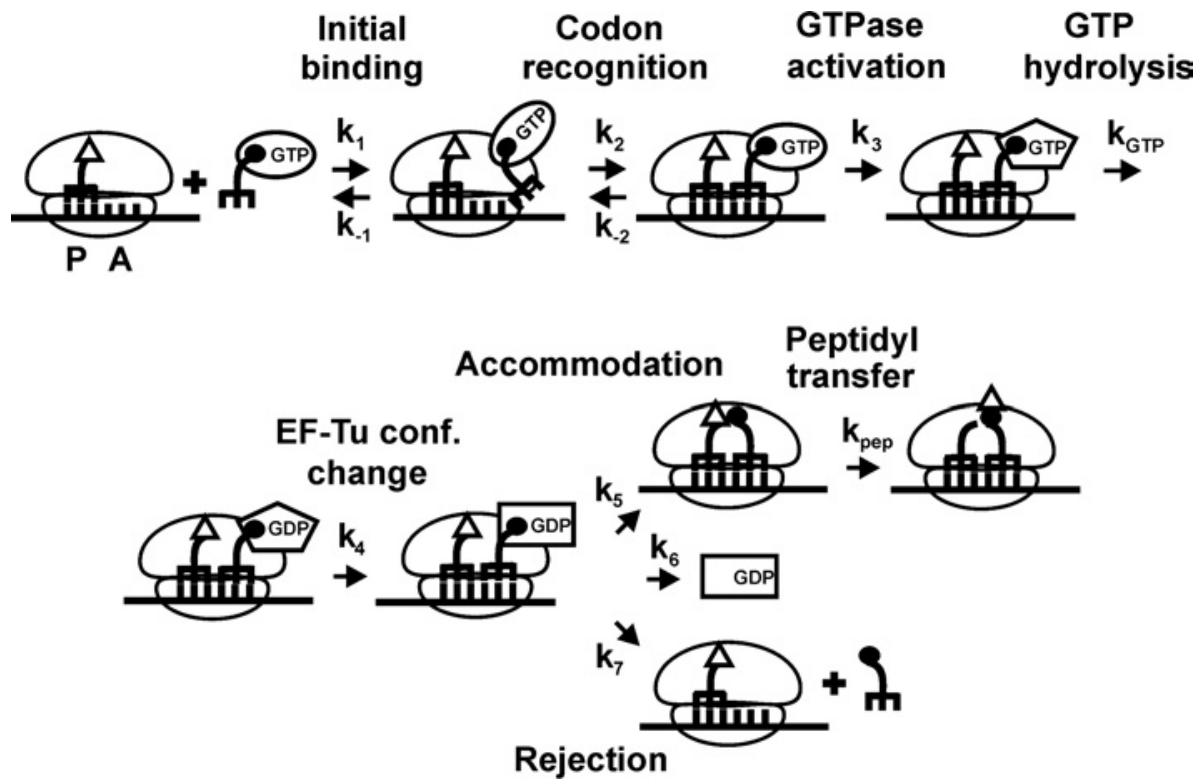

hydrolysis of cognate compared with near-cognate substrate [15].

The importance of induced fit for tRNA discrimination that was revealed by the kinetic analysis was confirmed by structural data. Comparison of the crystal structures of the $30 \mathrm{~S}$ subunits with and without an anticodon stem-loop fragment of tRNA bound to the A site has revealed that the formation of the cognate codon-anticodon duplex induces a conformational change in the decoding region that involves the conserved bases A1492, A1493, G530 and C1054 of 16S rRNA. These bases change position and form A-minor interactions in the minor groove of the first two base-pairs of the codon-anticodon complex. A-minor interactions are sequence-independent, but require Watson-Crick geometry. A1493 binds in the minor groove of the first base-pair. G530 and A1492, together with C518 and the ribosomal protein $\mathrm{S} 12$, act in a concerted way to monitor the second base-pair. At the third position, the codon interacts with G530, and it interacts indirectly through an $\mathrm{Mg}^{2+}$ ion with C518 and protein S12, whereas C1054 interacts with nucleotide 36 of tRNA $[4,16,17]$.

Further evidence for the importance of induced fit for aa-tRNA selection has come from experiments with the antibiotics paromomycin and streptomycin, both of which induce misreading. Kinetic results indicate that paromomycin switches the ribosome into a high-affinity/high-activity conformation, regardless of whether cognate or near-cognate aa-tRNA is bound to the A site [18]. Paromomycin binds to $16 \mathrm{~S}$ rRNA in the decoding centre [19] and induces (i) a local conformational change that is similar to, and enhanced by, the change induced by cognate codon recognition and (ii) a global rearrangement of the $30 \mathrm{~S}$ subunit that results in closure of the decoding site $[16,17]$. Streptomycin binds to several regions of $16 \mathrm{~S}$ rRNA and makes a contact with protein S12, thereby restricting internal movements within the $30 \mathrm{~S}$ subunit [19]. Streptomycin stabilized the binding of both cognate and near-cognate ternary complexes, whereas the effect on GTP hydrolysis was opposite for cognate and near-cognate codon-anticodon complexes, resulting in almost identical rate constants of GTP hydrolysis on cognate and near-cognate codons. This suggests that streptomycin interferes with transmission of the conformational changes within or near the $30 \mathrm{~S}$ decoding centre to the functional centres on the $50 \mathrm{~S}$ subunit, thereby trapping the ribosome in a conformation that is slow in signal transmission and unselective.

In conclusion, important conformational changes are induced by the fit between the mRNA codon and the anticodon of tRNA: conformational change in the decoding region, mobility of the $30 \mathrm{~S}$ subunit and those rearrangements that increase the forward rate constants of steps limiting the chemical steps, in particular GTPase activation and aa-tRNA accommodation in the A site. These conformational changes are different for cognate and near-cognate aa-tRNAs and lead to differences in the rate of forward reactions, which provide the major contribution to the discrimination against incorrect substrates by the ribosome.

\section{Peptide-bond formation: the ribosome as an entropy trap}

The PT centre of the ribosome is located on the $50 \mathrm{~S}$ subunit in domain V of $23 \mathrm{~S}$ rRNA. Crystal structures showed that the 
Table 1 | Activation parameters for the second-order uncatalysed $\left(k_{\text {non }}\right)$ and the ribosome-catalysed peptide bond formation as a second-order (limiting puromycin concentration, $k_{\text {cat }} / K_{M}$ ) or first-order (saturating puromycin, $k_{\text {cat }}$ ) reaction

The efficiency of the ribosome as a catalyst is given by the ratio of $k_{\text {cat }} / K_{M}$ to $k_{\text {non. }}$. Data are from [26].

\begin{tabular}{|c|c|c|c|c|}
\hline \multirow[b]{2}{*}{ Reaction } & \multirow[b]{2}{*}{ Rate constant } & \multicolumn{3}{|c|}{ Activation parameters $(\mathrm{kcal} / \mathrm{mol})$ at $25^{\circ} \mathrm{C}$} \\
\hline & & $\Delta G \neq$ & $\Delta H^{\neq}$ & $I \Delta S^{\neq}$ \\
\hline Uncatalysed & $k_{\text {nоก }}=3 \times 10^{-4} \mathrm{M}^{-1} \cdot \mathrm{s}^{-1}$ & 22.2 & 9.1 & -13.1 \\
\hline Catalysed (second order) & $k_{\text {cat }} / K_{M}=10^{3} M^{-1} \cdot s^{-1}$ & 14.0 & 16.0 & 2.0 \\
\hline Catalysed (first order) & $k_{\text {cat }}=5 s^{-1}$ & 16.5 & 17.2 & 0.7 \\
\hline
\end{tabular}

PT centre consists exclusively of RNA [20-24], indicating that the ribosome is a ribozyme. The rate of peptidebond formation on the ribosome is $50 \mathrm{~s}^{-1}$ or higher [25]. The rate of the uncatalysed reaction was estimated to be $3 \times 10^{-4} \mathrm{M}^{-1} \cdot \mathrm{s}^{-1}$, based on the rates observed in model reactions where the aminolysis of the ethylene glycol ester of $N$-formylglycine by a primary amine was studied. This value can be compared with the second-order rate constant $k_{\text {cat }} / K_{\mathrm{M}}=1000 \mathrm{M}^{-1} \cdot \mathrm{s}^{-1}$ for the reaction on the ribosome [26]. Thus, on the ribosome, the reaction is accelerated more than $10^{6}$-fold.

The catalytic mechanism of peptide-bond formation on the ribosome is not known in detail. At the PT centre, the amino group of aa-tRNA bound at the A site attacks the ester linkage between the carboxylate group of the growing peptide chain and the $3^{\prime} \mathrm{OH}$ group of pept-tRNA bound at the $\mathrm{P}$ site. The ribosome may contribute to catalysis in a number of ways: by substrate positioning, by electrostatic stabilization of the transition state or by taking part in proton transfer steps during the reaction (general acid/base catalysis). The rate of peptide-bond formation is known to be inhibited approx. 100 -fold by the protonation of a ribosomal group with $\mathrm{p} K_{\mathrm{a}}=7.5$ [25]. That observation would be consistent with general acid/base catalysis and/or a $\mathrm{pH}$-dependent conformation change within the active site. On the basis of the crystal structure, a chemical role in catalysis was originally ascribed to a highly conserved adenine residue (A2451 in Escherichia coli), and a charge relay system involving G2447 was postulated to bring about the required $\mathrm{p} K_{\mathrm{a}}$ shift of A2451 [21]. However, mutational analysis showed that replacing A2451 with other bases slowed down the PT rate only 100 -fold $[25,27,28]$. Furthermore, substitution of G2447 was found to have no effect on the ionization of the ribosome group with $\mathrm{p} K_{\mathrm{a}}=7.5$ [29]. Mutations of four universally conserved nucleotides in the innermost layer of the active site, A2451, U2506, U2585 and A2602, substantially decreased the rates of peptide-bond formation using puromycin as the A-site substrate, whereas the rates were unaffected when aa-tRNAs were used [30]. These results do not support a charge relay mechanism involving G2447, with A2451 acting as general base. Possible participation of a ribosomal group with $\mathrm{p} K_{\mathrm{a}}<5$ could not be examined since ribosomes lose activity at $\mathrm{pH}<5.2$.

Comparison of the activation parameters of peptide-bond formation (Table 1) indicated that the activation enthalpy for the reaction on the ribosome $(16 \mathrm{kcal} / \mathrm{mol} ; 1 \mathrm{kcal} \approx$ $4184 \mathrm{~J}$ ) is more unfavourable compared with that for model substrates in solution $(9.1 \mathrm{kcal} / \mathrm{mol})$, which is the opposite of what one could expect for the general acid/base catalysis. However, the activation entropy for peptidyl transfer within the ribosome $(+2.0 \mathrm{kcal} / \mathrm{mol})$ is much more favourable compared with that for ester aminolysis in solution $\left(-13.1 \mathrm{kcal} / \mathrm{mol}\right.$ at $\left.25^{\circ} \mathrm{C}\right)$. The difference in entropy is sufficient to account for the $4 \times 10^{6}$-fold rate enhancement produced by the ribosome. The favourable activation entropy may arise either from the positioning of substrates in the active site or from the removal of the reaction from bulk water. It has been shown that proximity effects alone, in aqueous solution, do not produce a rate enhancement comparable with that achieved by the ribosome [31]. These findings suggest that general acid/base catalysis does not play a significant role in peptidyl transfer in the ribosome. Instead, the observed $\mathrm{pH}$ dependence of the rate constant for peptide-bond formation [25] may arise from conformational changes in the PT centre. Consistent with that possibility, $\mathrm{pH}$-dependent rearrangements of $23 \mathrm{~S}$ rRNA at the active site have been demonstrated [32].

\section{Iranslocation: is EF-G a deterministic or a Brownian motor?}

The last step of the elongation cycle is translocation. It entails the synchronous movement of two tRNAs and the mRNA on the ribosome: deacylated tRNA moves from the $\mathrm{P}$ site to the $\mathrm{E}$ site and pept-tRNA moves from the A site to the $\mathrm{P}$ site. Translocation is catalysed by EF-G, which hydrolyses GTP during the reaction. Rapid kinetic and biochemical analysis has established the following reaction sequence (Figure 2 and Table 2) [33,34]: binding of EF-G. GTP to the pretranslocation complex triggers rapid GTP hydrolysis (unbound EF-G has no measurable GTPase activity). Ribosomal residues involved in GTPase activation include L7/L12 protein and probably also elements of $23 \mathrm{~S}$ rRNA, such as the sarcin-ricin loop [35]. GTP hydrolysis appears to induce a conformational change of the factor that is coupled with a rearrangement of the ribosome ('unlocking') that precedes, and limits the rates of, subsequent tRNAmRNA movement and $P_{i}$ release from EF-G $\cdot G D P \cdot P_{i}[34]$. Ribosome unlocking is impaired in the absence of GTP hydrolysis $[34,36]$. The process of translocation is completed by $\mathrm{P}_{\mathrm{i}}$ release, which leads to further conformational changes 
Figure 2 | Kinetic model of translocation

Ribosomes are depicted in two conformations, closed and open, to indicate unlocking. EF-G is depicted in different conformations and orientations on the ribosome, based on models from cryo-electron microscopy [38]. Rate constants are from [34] (upper row) and B. Wilden, M.V. Rodnina and W. Wintermeyer, unpublished work (lower row).
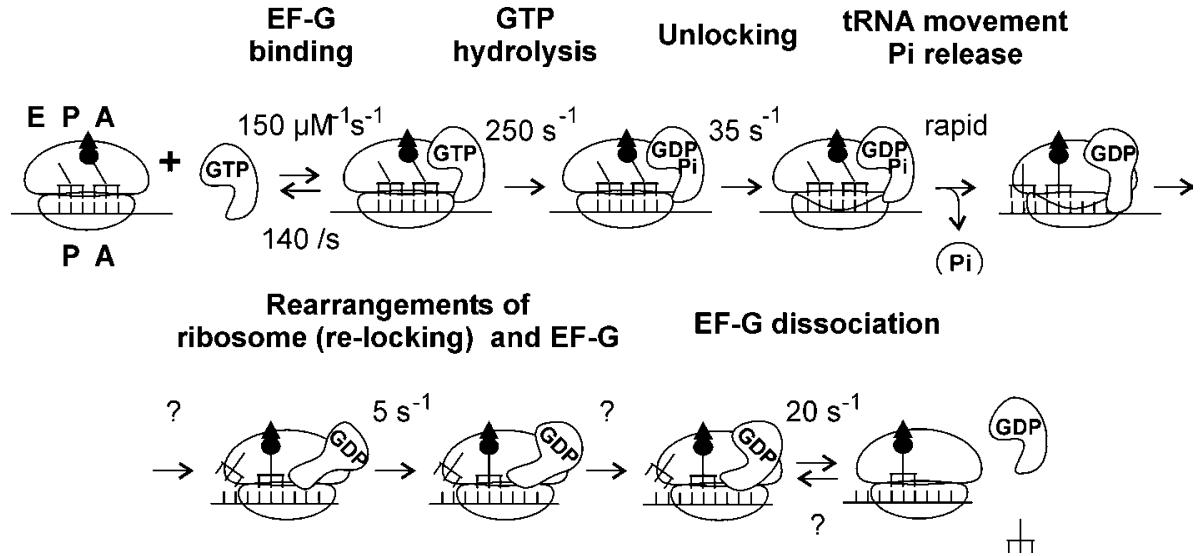

Table 2 | Fidelity-determining rate constants and efficiency of initial selection steps and proofreading steps $[14,15]$ n.d., not determined.

\begin{tabular}{|c|c|c|c|c|c|c|}
\hline & \multicolumn{4}{|c|}{ Rate constants $\left(\mathrm{s}^{-1}\right)$} & \multicolumn{2}{|l|}{ Selectivity } \\
\hline & $k_{-2}$ & $k_{3}$ & $k_{7}$ & $k_{5}$ & Initial selection & Proofreading \\
\hline \multicolumn{7}{|l|}{ Low-accuracy conditions } \\
\hline Cognate codon & 0.2 & 500 & $<0.3$ & 6 & 1.1 & 60 \\
\hline Near-cognate codon & 17 & 50 & 6 & 1 & 1.1 & 60 \\
\hline \multicolumn{7}{|l|}{ High-accuracy conditions } \\
\hline Cognate codon & 0.2 & 260 & $<0.3$ & 7 & 60 & 15 \\
\hline Near-cognate codon & 80 & 0.4 & n.d. & n.d. & 60 & 15 \\
\hline
\end{tabular}

and, finally, dissociation of EF-G.GDP and deacylated tRNA from the ribosome.

According to the kinetic analysis, both tRNA-mRNA movement and $P_{i}$ release are intrinsically rapid and take place at random, implying that movement is not directly coupled with ribosome unlocking. This is corroborated by the observation that movement and $\mathrm{P}_{\mathrm{i}}$ release can be uncoupled in both directions by inhibiting either movement or $\mathrm{P}_{\mathrm{i}}$ release. For instance, movement, but not $\mathrm{P}_{\mathrm{i}}$ release, is blocked by antibiotics that bind in the $30 \mathrm{~S}$ decoding region, such as viomycin, paromomycin or hygromycin $\mathrm{B}$ $([33,37]$ and F. Peske, M.V. Rodnina and W. Wintermeyer, unpublished work). A point mutation at the tip of domain 4 of EF-G, H583K (His-583 $\rightarrow$ Lys), has the same effect [37]. Conversely, a point mutation in the ribosomal protein L7/12 inhibits $P_{i}$ release and has no effect on tRNA movement [34]. In summary, these results indicate that actual tRNA movement takes place after the unlocking rearrangement. Inhibition of movement by antibiotics binding in the decoding region suggests that, after unlocking, conformational changes of the $30 \mathrm{~S}$ subunit are required for movement. The effect of the $\mathrm{H} 583 \mathrm{~K}$ mutation indicates that the contact of domain 4 of EF-G with the shoulder region of the $30 \mathrm{~S}$ subunit in the pretranslocation complex, as revealed by cryo-electron microscopy [38], is involved in the opening up of the decoding region. As to actual movement, the kinetic results indicate that it takes place by diffusion, rather than being coupled with the unlocking rearrangement of the ribosome. The directionality of diffusion seems to follow the thermodynamic gradient, because the binding affinity of pept-tRNA is higher in the $\mathrm{P}$ site than in the A site. Additionally, EF-G movement accompanies the displacement of tRNA in such a way that, in the post-translocation state, domain 4 of EF-G occupies the 30S A site [38-40], thus effectively preventing back movement of pept-tRNA.

An important unresolved question concerns the driving force for the acceleration of movement during translocation. Is EF-G an active motor that couples the energy of GTP hydrolysis with the movement through direct mechanochemical coupling, or is it a Brownian motor that biases thermal fluctuations towards movement by structural anisotropy produced by GTP cleavage? Recently, substantial progress has been made in understanding the movement in different motor-like systems and the physics of mechanochemical systems in general [41]. The common theme is that motor proteins may generate force and vectorial motions by 
rectifying thermal fluctuations. In such Brownian motor models, chemical energy does not produce force by direct mechanochemical coupling. Instead, the motor diffuses along its track by random walk driven by Brownian motion, and the conformation induced by ATP hydrolysis merely biases the walk such that steps are more probable in the forward direction than in the backward direction $[42,43]$.

There are, in fact, parallels between deterministic motors, e.g. myosin and EF-G. In both cases, the products of nucleoside triphosphate hydrolysis, $\mathrm{ADP} \cdot \mathrm{P}_{\mathrm{i}}$ or GDP $\cdot \mathrm{P}_{\mathrm{i}}$, are trapped in the active site, and the protein adopts a strongly bound conformation before movement. However, if EF-G were a deterministic motor, GTP hydrolysis is expected to be coupled with the movement through release of $\mathrm{P}_{\mathrm{i}}$. Such a model of EF-G function is not favoured for a number of reasons. First, as discussed above, rapid translocation requires GTP hydrolysis, but not $P_{i}$ release, indicating indirect coupling between chemical and mechanical steps [34]. Furthermore, translocation can occur spontaneously without EF-G, albeit very slowly, or with EF-G but without GTP hydrolysis, i.e. under conditions where movement is due to thermal motion only $[33,36,44]$. These facts are more easily reconciled with a scenario in which EF-G utilizes the energy of GTP hydrolysis to induce a rearrangement of the ribosome and, subsequently (presumably driven by $\mathrm{P}_{\mathrm{i}}$ release), biases forward movement, which itself is spontaneous. Thus, we favour a model (which, in part, is speculative so far) in which EF-G has two functions. One function, performed by EF$\mathrm{G} \cdot \mathrm{GDP} \cdot \mathrm{P}_{\mathrm{i}}$, is to impose conformational strain on the ribosome to promote unlocking, which is a prerequisite for tRNA movement. The second is to bias diffusion to produce forward movement. The first function is driven by GTP hydrolysis directly, i.e. it can be referred to as mechanochemical function, whereas the other involves a reorientation of EF-G.GDP, resembling the function of a Brownian ratchet. Further mechanistic work, including force-velocity measurements on single translocating ribosomes, will be required to resolve completely these issues.

We acknowledge collaborations with V.I. Katunin and Yu.P. Semenkov (Russian Academy of Sciences), S.A. Strobel (Yale University) and R. Wolfenden and A. Sievers (University of North Carolina, Chapel Hill). Work in our laboratories is supported by the Deutsche Forschungsgemeinschaft, the European Union, the Alfried Krupp von Bohlen und Halbach-Stiftung, the Bundesministerium für Bildung und Forschung and the Fonds der Chemischen Industrie.

\section{References}

1 Gualerzi, C.O., Brandi, L., Caserta, E., Garofalo, C., Lammi, M.

La Teana, A., Petrelli, D., Spurio, R., Tomsic, J. and Pon, C.L. (2001) Cold Spring Harb. Symp. Quant. Biol. 66, 363-376

2 Moore, P.B. and Steitz, T.A. (2003) Annu Rev. Biochem. 72, 813-850

3 Nakamura, Y. and Ito, K. (2003) Trends Biochem. Sci. 28, 99-105

4 Ogle, J.M., Carter, A.P. and Ramakrishnan, V. (2003) Trends Biochem. Sci. 28, 259-266

5 Ramakrishnan, V. (2002) Cell (Cambridge, Mass.) 108, 557-572
6 Steitz, T.A. and Moore, P.B. (2003) Trends Biochem. Sci. 28 411-418

7 Yonath, A. (2002) Annu. Rev. Biophys. Biomol. Struct. 31, 257-273

8 Yonath, A. (2003) Biol. Chem. 384, 1411-1419

9 Frank, J. (2000) Chem. Biol. 7, R133-R141

10 Frank, J. (2003) Biopolymers 68, 223-233

11 Rodnina, M.V. and Wintermeyer, W. (2001) Annu. Rev. Biochem. 70, 415-435

12 Rodnina, M.V. and Wintermeyer, W. (2001) Trends Biochem. Sci. 26, 124-130

13 Rodnina, M.V., Daviter, T., Gromadski, K. and Wintermeyer, W. (2002) Biochimie 84, 745-754

14 Pape, T., Wintermeyer, W. and Rodnina, M.V. (1999) EMBO J. 18 3800-3807

15 Gromadski, K.B. and Rodnina, M.V. (2004) Mol. Cell 13, 191-200

16 Ogle, J.M., Murphy, F.V., Tarry, M.J. and Ramakrishnan, V. (2002) Cell (Cambridge, Mass.) 111, 721-732

17 Ogle, J.M., Brodersen, D.E., Clemons, Jr, W.M., Tarry, M.J., Carter, A.P. and Ramakrishnan, V. (2001) Science 292, 897-902

18 Pape, T., Wintermeyer, W. and Rodnina, M.V. (2000) Nat. Struct. Biol. 7 104-107

19 Carter, A.P., Clemons, Jr, W.M., Brodersen, D.E., Morgan-Warren, R.J., Wimberly, B.T. and Ramakrishnan, V. (2000) Nature (London) 407, 340-348

20 Ban, N., Nissen, P., Hansen, J., Moore, P.B. and Steitz, T.A. (2000) Science 289, 905-920

21 Nissen, P., Hansen, J., Ban, N., Moore, P.B. and Steitz, T.A. (2000) Science 289, 920-930

22 Schlunzen, F., Zarivach, R., Harms, J., Bashan, A., Tocilj, A., Albrecht, R., Yonath, A. and Franceschi, F. (2001) Nature (London) 413, 814-821

23 Harms, J., Schluenzen, F., Zarivach, R., Bashan, A., Gat, S., Agmon, I. Bartels, H., Franceschi, F. and Yonath, A. (2001) Cell (Cambridge, Mass.) 107, 679-688

24 Hansen, J.L., Schmeing, T.M., Moore, P.B. and Steitz, T.A. (2002) Proc. Natl. Acad. Sci. U.S.A. 99, 11670-11675

25 Katunin, V.I., Muth, G.W., Strobel, S.A., Wintermeyer, W. and Rodnina, M.V. (2002) Mol. Cell 10, 339-346

26 Sievers, A., Beringer, M., Rodnina, M.V. and Wolfenden, R. (2004) Proc. Natl. Acad. Sci. U.S.A. 101, 7897-7901

27 Polacek, N., Gaynor, M., Yassin, A. and Mankin, A.S. (2001) Nature (London) 411, 498-501

28 Thompson, J., Kim, D.F., O'Connor, M., Lieberman, K.R., Bayfield, M.A., Gregory, S.T., Green, R., Noller, H.F. and Dahlberg, A.E. (2001) Proc. Natl. Acad. Sci. U.S.A. 98, 9002-9007

29 Beringer, M., Adio, S., Wintermeyer, W. and Rodnina, M. (2003) RNA 9 919-922

30 Youngman, E.M., Brunelle, J.L., Kochaniak, A.B. and Green, R. (2004) Cell (Cambridge, Mass.) 117, 589-599

31 Tamura, K. and Schimmel, P. (2001) Proc. Natl. Acad. Sci. U.S.A. 98 1393-1397

32 Bayfield, M.A., Dahlberg, A.E., Schulmeister, U., Dorner, S. and Barta, A. (2001) Proc. Natl. Acad. Sci. U.S.A. 98, 10096-10101

33 Rodnina, M.V., Savelsbergh, A., Katunin, V.I. and Wintermeyer, W. (1997) Nature (London) 385, 37-41

34 Savelsbergh, A., Katunin, V.I., Mohr, D., Peske, F., Rodnina, M.V. and Wintermeyer, W. (2003) Mol. Cell 11, 1517-1523

35 Mohr, D., Wintermeyer, W. and Rodnina, M.V. (2002) Biochemistry 41, $12520-12528$

36 Katunin, V.I., Savelsbergh, A., Rodnina, M.V. and Wintermeyer, W. (2002) Biochemistry 41, 12806-12812

37 Savelsbergh, A., Matassova, N.B., Rodnina, M.V. and Wintermeyer, W. (2000) J. Mol. Biol. 300, 951-961

38 Stark, H., Rodnina, M.V., Wieden, H.-J., van Heel, M. and Wintermeyer, W. (2000) Cell (Cambridge, Mass.) 100, 301-309

39 Frank, J. and Agrawal, R.K. (2000) Nature (London) 406, 318-322

40 Agrawal, R.K., Penczek, P., Grassucci, R.A. and Frank, J. (1998) Proc. Natl. Acad. Sci. U.S.A. 95, 6134-6138

41 Keller, D. and Bustamante, C. (2000) Biophys. J. 78, 541-556

42 Astumian, R.D. (1997) Science 276, 917-922

43 Okada, Y. and Hirokawa, N. (1999) Science 283, 1152-1157

44 Noller, H.F., Yusupov, M.M., Yusupova, G.Z., Baucom, A. and Cate, J.H. (2002) FEBS Lett. 514, 11-16

Received 13 July 2004 